\title{
The evolution of knee anterior cruciate ligament tear incidence in a single ski resort: 23 years overview
}

\begin{abstract}
Objective: This study aims to describe the evolution of knee anterior cruciate ligament (ACL) incidence per year in a single ski resort.

Methods: A Cross-sectional study was designed. The injury data were collected under the same conditions since 1992, from the single medical centre at the ski resort. Staff doctors collected all data related to diagnosis and mechanisms of injury. Spearman correlation was used to determine if the injury rate was increasing yearly, using a significance level of 0.05 .

Results: The overall injury rate was $3.52 / 1000$ visitors $(3.52 \%$ ) per year. Meanwhile, the ACL rate and other knee ligament injury rates were $0.36 / 1000$ visitors $(0.36 \%$ o and $0.86 / 1000$ visitors $(0.86 \%$ ) per year, respectively. The rate of other knee ligament injuries had been significantly decreasing over time (rho $-0.54, \mathrm{p}=0.01$ ), but ACL tear rate has not (rho $-0.25, \mathrm{p}=0.26$ ). The mean age at the time of injury has been increasing significantly over the years, in both ACL tear and OKL injuries (rho 0.82, p<0.00 and rho $0.75, \mathrm{p}<0.00$ respectively).

Conclusion: Knee ligament injuries are a frequent snow sport related injury, representing in this review $24 \%$ of all lesions. The ACL tear rate did not change over the duration of this study, despite the overall decrease in other knee ligament injuries. Evolution and technology in skiing and snowboarding have not achieved a decrease in ACL tear rate.
\end{abstract}

Keywords: knee ligament injuries, ACL injury, ski injuries, snowboard injuries
Volume 2 Issue 3 - 2018

\author{
Maximiliano Barahona,' José Tomás Rojas,' \\ Carolina Courtin,' Sergio Aguirre, ${ }^{2}$ Aleix \\ Vidal $^{2}$ \\ 'Hospital Clínico Universidad de Chile, Chile \\ ${ }^{2}$ Centro Medico Baqueira, Spain
}

\begin{abstract}
Correspondence: Barahona Maximiliano, Orthopaedic surgeon, Hospital Clinico Universidad de Chile, Chile, Email maxbarahonavasquez@gmail.com
\end{abstract}

Received: May 16, 2018 | Published: May 29, 2018

\section{Introduction}

Both, alpine skiing and snowboarding are popular winter sports around the world. They gather 11.5 million athletes in the United States of America (USA) and almost 200 million athletes over the world every year. The participation in these activities, as a hobby or in a professional way, imply an exposure to falls and high-speed collisions that lead to a high rate of traumatic injuries. ${ }^{1,2}$ Knee injuries are one of the most common injuries, ACL tear accounting nearly $50 \%$ of them $;{ }^{3}$ most of this injuries occurring when the athlete is still skiing, being turning or landing from a jump. ${ }^{4}$ Winter sports are continually changing. Technological changes in boots, fixations and ski types, along with changes in the styles practiced could imply a change in the epidemiology of knee ligament injuries. ${ }^{1,5}$ Numerous studies describe the epidemiology of knee injuries when skiing and snowboarding; however, there is very little evidence describing the evolution of anterior cruciate ligament (ACL) incidence over time. ${ }^{6-8}$ This study aims to describe the evolution of knee anterior cruciate ligament (ACL) incidence per year in a single ski resort.

\section{Methods}

A descriptive study was designed which our institutional ethics review board approved. Injury data of a single medical centre ski resort was used. Data was collected between 1992 and 2015 using the same methodology. Mainly, two types of knee lesions were recorded because of their relatively high prevalence: ACL tears and "other ligament knee injury" being those: isolated medial collateral ligament (MCL) grade 1-3, isolated posterior cruciate ligament (PCL) grade 1-2 and lateral collateral ligament (LCL) grade 1-2. Sports physicians registered all data after a clinical exam and imaging study (X-ray and magnetic resonance). Demographic data was also recollected. The number of visitors per season was obtained from the ski resort data; nevertheless, it was not possible to obtain the age and gender of each visitor. Injury rate by I season (I is the season year) was calculated as follows.

$$
\begin{gathered}
; \text { Total injury rate }(i)=[(\text { number of injury }(i) / \text { visitors }(i)) * 1000] \\
\text { total knee injury rate }(i)=[(\{\text { ACL tear }(i)+\text { other ligament }(i)\} \text { visitors }(i)) * 1000] \\
\text { ACL injury rate }(i)=[(A C L \text { tear }(i) / \text { visitors }(i)) * 1000] ; \\
\text { other ligament injury }=[\text { other ligament injury } / \text { visitors }) * 1000], \\
; \text { female ACL injury rate }(i)=[(\text { female ACL tear }(i) / \text { visitors }(i)) * 1000] \\
\text { male ACL injury rate }(i)=[(\text { Male ACL tear }(i) / \text { visitors }(i)) * 1000]
\end{gathered}
$$

Spearman correlation (Rho) was calculated to analyze the behaviour of the injury incidence over the years. A positive rho means that the injury rate increased; meanwhile, a negative rho means that the injury rate was decreasing over the years. All Rho were analysed using a null hypothesis that $\mathrm{rho}=0$ (no correlation meaning that injury 
rate has not changed among years). For comparing the ACL injury rate between genders, a non-parametric median test for unpaired data was used. A significant level of 0.05 was used. All analyses were performed using Stata v11.2 (StataCorp LP, College Station, Texas, USA).

\section{Results}

The overall injury rate per year was $3.52 / 1000$ visitors (3.52\%o). The overall anterior cruciate ligament injury rate was $0.36 / 1000$ visitors per year $(0.36 \%)$; meanwhile, other knee ligament injury rates were $0.86 / 1000$ visitors per year $(0.86 \%)$. The rate of other knee ligament injuries has been significantly decreasing over time (rho $-0.54, \mathrm{p}=0.01$ ), but ACL tear rate has not (rho $-0.25, \mathrm{p}=0.26$ ) (Figure 1). The major ACL injury rate was achieved in 1992-1993 and 1999-1999 were the rate increased above $0.5 / 1000$ visitors $(0.5 \%)$; meanwhile, the lowest
ACL injury rate was achieved in 2010/2011 season where the rate decreased under $0.2 / 1000$ visitors $(0.2 \%)$. The mean ACL tear rate among the 23 seasons was $0.35 / 1000(35 \%$ ) in ski, while $0.01 / 1000$ $(0.01 \%)$ in snowboard. No significant variation over time was found, reaching $\mathrm{rho}=-0.26 \mathrm{rho}=0.16$ respectively. Unfortunately, the number of users of ski or snowboard is not registered, so the indicator is by the number of visitors. Mean age by year range between 35 to 40 years old. The 1993-1994 season achieved the younger mean age reaching 35 years old, on the other hand, the 2007-2008 season and the 20092010 season achieved the older mean age, being 40 years. The mean age at the time of injury has been increasing significantly over the years, in both ACL tear and other knee ligament injuries (rho 0.82, $p<0.00$ and rho $0.75, p<0.00$ respectively). ACL injury rate was higher in female gender $(\mathrm{p}<0.00)$, nevertheless the rate does not vary among years in both genders, achieving rho $-0.23(\mathrm{p}=0.28)$ for male and rho $-0.19(\mathrm{p}=0.38)$ for women (Table 1)(Figure 2).

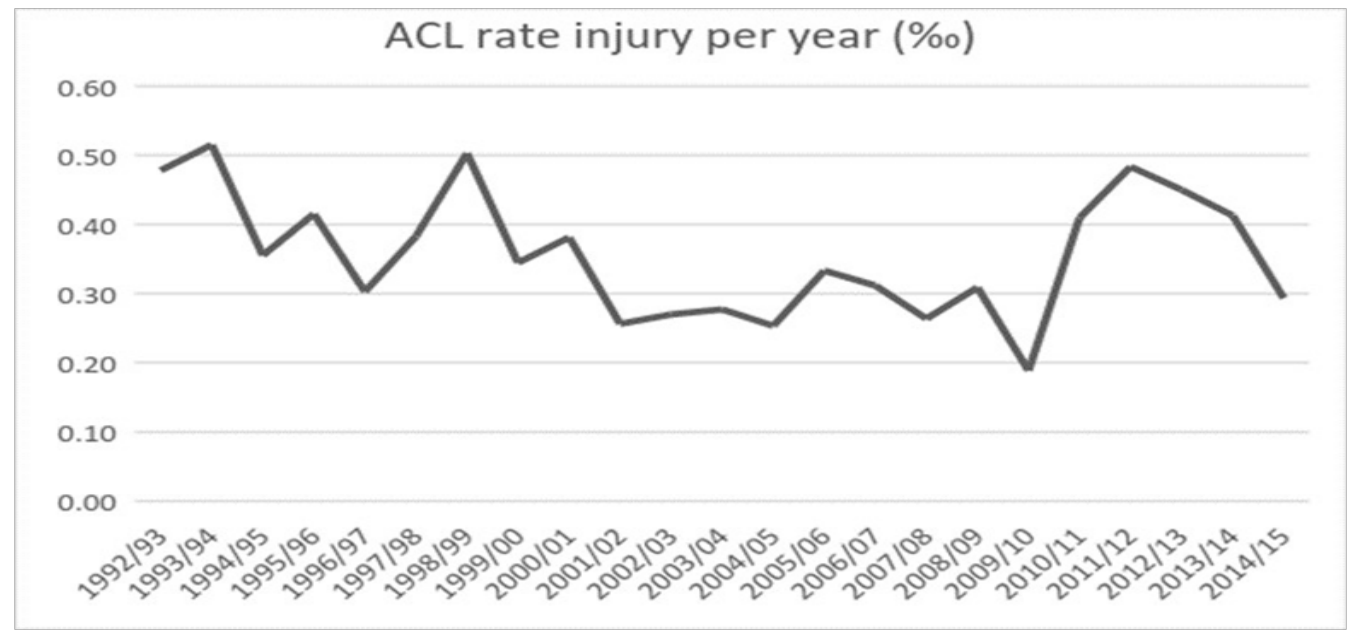

Figure I Shows $A C L$ injury rate per 1000 visitors per year. $A C L$ tear has not changed over the period analysed in this study (rho $-0.25, p=0.26)$.

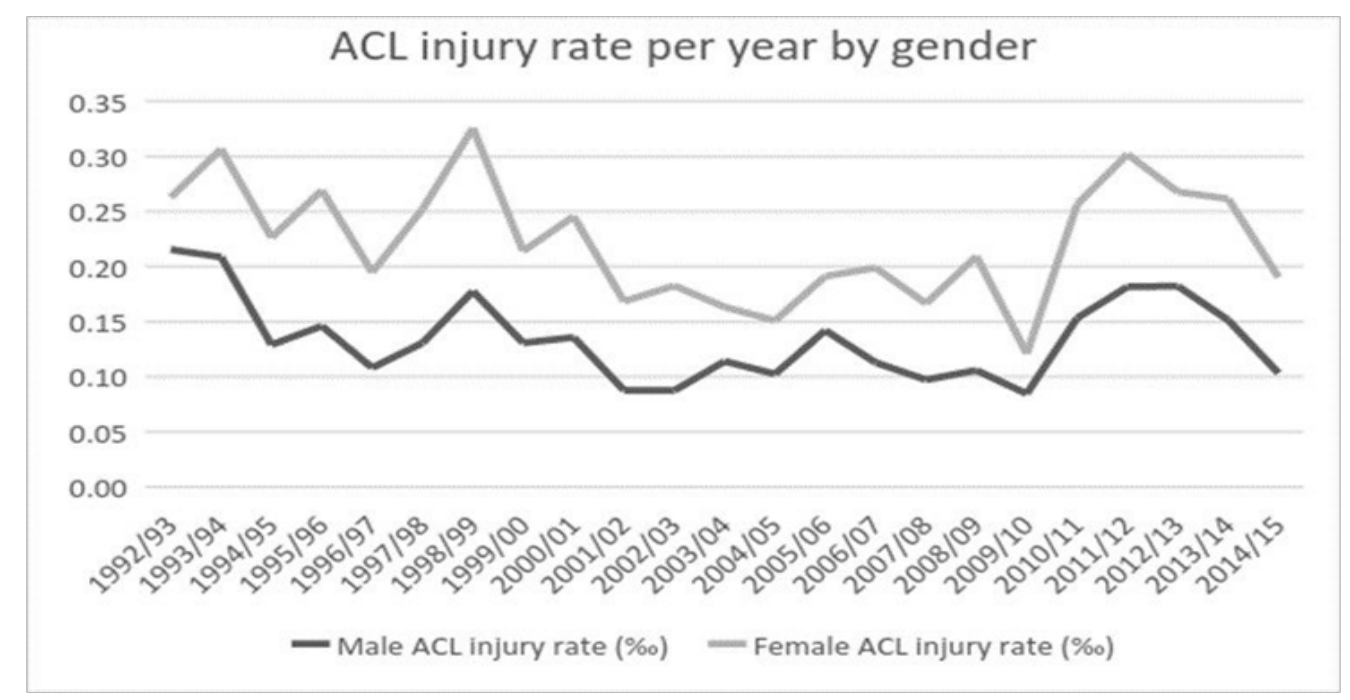

Figure 2 Shows $A C L$ injury rate per 1000 visitors per years by gender. Male gender has a lower rate of ACL injury. Both, Male and Female rate has not significantly changed between 1992 and 2015. 
Table I Shows ACL injury rate per year per 1000 visitors. The male and female rate was calculated using the total visitors. Over the years, the injury rate was significantly higher in male than female $(p<0.00)$

\begin{tabular}{llll}
\hline Season & ACL rate injury (\%) & Male ACL injury rate (\%) & Female ACL injury rate (\%) \\
\hline $992 / 93$ & 0,48 & 0,22 & 0,26 \\
$1993 / 94$ & 0,52 & 0,21 & 0,31 \\
$1994 / 95$ & 0,36 & 0,13 & 0,23 \\
$1995 / 96$ & 0,42 & 0,15 & 0,27 \\
$1996 / 97$ & 0,30 & 0,11 & 0,19 \\
$1997 / 98$ & 0,38 & 0,13 & 0,25 \\
$1998 / 99$ & 0,50 & 0,18 & 0,33 \\
$1999 / 00$ & 0,34 & 0,13 & 0,21 \\
$2000 / 01$ & 0,38 & 0,14 & 0,25 \\
$2001 / 02$ & 0,26 & 0,09 & 0,17 \\
$2002 / 03$ & 0,27 & 0,09 & 0,18 \\
$2003 / 04$ & 0,28 & 0,11 & 0,16 \\
$2004 / 05$ & 0,25 & 0,10 & 0,15 \\
$2005 / 06$ & 0,33 & 0,14 & 0,19 \\
$2006 / 07$ & 0,31 & 0,11 & 0,20 \\
$2007 / 08$ & 0,26 & 0,10 & 0,17 \\
$2008 / 09$ & 0,31 & 0,11 & 0,21 \\
$2009 / 10$ & 0,19 & 0,08 & 0,12 \\
$2010 / 11$ & 0,41 & 0,15 & 0,26 \\
$2011 / 12$ & 0,48 & 0,18 & 0,30 \\
$2012 / 13$ & 0,45 & 0,18 & 0,27 \\
$2013 / 14$ & 0,41 & 0,10 & \\
$2014 / 15$ & 0,29 & & 0,19 \\
\hline
\end{tabular}

\section{Discussion}

This study shows that despite the emphasis to pursue preventive measures during these years, the rate of ACL injury per year has not decreased. ACL tear shows a higher incidence in ski than snowboard and athletes in the 3rd decade and female gender. The strengths of this study are that the data was collected for 23 years and those physicians with expertise in alpine ski and snowboard injuries made all records and that all the attention were done in the same centre, which is the only emergency department in this ski resort. Kroll et al. ${ }^{9}$ they propose different strategies to demonstrate changes in the prevalence of a sports injury. In our case, our strength was to increase the monitoring time, which allows us to follow a large number of subjects at risk. Limitations of this study are that it is retrospective and it misses data of total visitors by gender and sport (ski o snowboard). A recent meta-analysis shows an ACL injury incidence between 0.02 and 0.04 in highly experienced skiers and between 0.4-0.6 in inexperienced skiers. However, these numbers are not comparable as we used the number of visitors and Prodromos et al..$^{10}$ used the number of exposures as the denominator. Nevertheless, the rates tend to be similar. $^{10}$

The same study lacks to find a difference in gender as we found in this study..$^{10}$ It is necessary to notice that the rates by gender in our study, were not calculated by the gender of the visitors, so it is biased for our findings; nevertheless, the different ratio between gender is reported in many sports, especially basketball and soccer. Several risk factors have been identified to explain the high rate in female athletes; the most accepted are lower neuro balance skills, hormonal factors, a major incidence of dynamic valgus and a narrower intercondylar notch. ${ }^{11-14}$ Equipment plays a crucial role in reducing sport-related injury. For example, In 1997/1998 carving skis were introduced and in 2012-2013 the International Ski Federation (FIS) changes the regulation for the ski sidecut radius. ${ }^{5,15}$ Despite this efforts, this study shows that ACL tear rate per year did not change over the duration 
of this study, despite the overall decrease in other knee ligament injuries. Evolution and technology in skiing and snowboarding have not achieved a decrease in ACL tear rate. A novel biomechanical study shows that the greater the rear stiffness of the boot, the higher the tension in the anterior cruciate ligament while first $50 \mathrm{~ms}$ of landing, therefore decreasing the stiffness could prevent this injury. However, this is rejected by athletes given that they perceive a decrease in their overall performance. The authors conclude that work must be done on the counterpart, that is, to reduce the tension generated in the quadriceps and more hamstring activations in landing. ${ }^{16}$

Another critical issue is to control the trunk inclination during landing, ${ }^{17}$ so core strength seems to be critical to avoid ACL lesions. ${ }^{4,15}$ Taken this data into account, seems that nowadays more emphasis must be done in core and hamstring strength training than equipment development. ${ }^{5,18}$ Recently, neuromuscular warm-up strategy before resuming sport have gain popularity for their excellent results in sport-related injury prevention, this programs made emphasis in core control as well. ${ }^{19,20}$ To our knowledge, no study measuring neuromuscular training effect on sky or snowboarding related injury has been performed, but literature is plenty of good results in other sports. Many programs have shown promising results in ACL tear prevention like PEP and FIFA11. PEP has a risk ratio of 0.18 to have an ACL tear; meanwhile, FIFA11 has a risk ratio of 0.42 of having any knee injury. ${ }^{10,21,22}$ Reports show that neuromuscular injury prevention programs have an impact in about $70 \%$ in reducing ACL tear in highrisk population. ${ }^{15}$ Efforts must be made to develop a specific plan for snow sports

\section{Conclusion}

Knee ACL tear incidence in winter sport has not changed between 1992 to 2015 in winter sports, significant efforts in prevention must be performed with particular emphasis on neuro balance training which is a well-known modifiable risk factor.

\section{Acknowledgements}

The first author acknowledges Leonel Barahona for his unconditional support, love and motivation and we have no funding.

\section{Conflicts of interest}

Authors declare that they have no conflict of interest.

\section{References}

1. Alentorn GE, Myer GD, Silvers HJ, et al. Prevention of non-contact anterior cruciate ligament injuries in soccer players. Part 1: Mechanisms of injury and underlying risk factors. Knee Surg Sports Traumatol Arthrosc. 2009;17(7):705-729.

2. McBeth PB, Ball CG, Mulloy RH, et al. Alpine ski and snowboarding traumatic injuries: incidence, injury patterns and risk factors for 10 years. Am J Surg. 2009;197(5):560-563.

3. Stevenson H, Webster J, Johnson R, et al. Gender differences in knee injury epidemiology among competitive alpine ski racers. Iowa Orthop J. 1998;18:64-66.

4. Bere T, Bahr R. Injury prevention advances in alpine ski racing: harnessing collaboration with the International Ski Federation (FIS), long-term surveillance and digital technology to benefit athletes. Br J Sports Med. 2014;48(9):738.
5. Kim S, Endres NK, Johnson RJ, et al. Snowboarding injuries: trends over time and comparisons with alpine skiing injuries. The Am J Sports Med. 2012;40(4):770-776.

6. Hughes G, Watkins J. A risk-factor model for anterior cruciate ligament injury. Sports Med. 2006;36(5):411-428.

7. Stenroos A, Handolin L. Incidence of Recreational Alpine Skiing and Snowboarding Injuries: six years experience in the largest ski resort in Finland. Scand J Surg. 2015;104(2):127-131.

8. Yu B, Garrett WE. Mechanisms of non-contact ACL injuries. Br J Sports Med. 2007;41 Suppl 1:i47-51.

9. Kröll J, Spörri J, Steenstrup SE, et al. How can we prove that a preventive measure in elite sport is effective when the prevalence of the injury (eg, ACL tear in alpine ski racing) is low? A case for surrogate outcomes. $\mathrm{Br} J$ Sports Med. 2017;51(23):1644-1645.

10. Prodromos CC, Han Y, Rogowski J, et al. A meta-analysis of the incidence of anterior cruciate ligament tears as a function of gender, sport and a knee injury-reduction regimen. Arthroscopy. 2007;23(12):1320-1325.

11. Henry JC, Kaeding C. Neuromuscular differences between male and female athletes. Curr Womens Health Rep. 2001;1(3):241-244.

12. Hewett TE, Myer GD, Ford KR. Anterior cruciate ligament injuries in female athletes. Am J Sports Med. 2006;34(2):299-311.

13. Sutton KM, Bullock JM. Anterior cruciate ligament rupture: differences between males and females. J Am Acad Orthop Surg. 2013;21(1):41-50.

14. Wild CY, Steele JR, Munro BJ. Why do girls sustain more anterior cruciate ligament injuries than boys?: a review of the changes in estrogen and musculoskeletal structure and function during puberty. Sports Med. 2012;42(9):733-749.

15. Jordan MJ, Aagaard P, Herzog W. Anterior cruciate ligament injury/ reinjury in alpine ski racing: a narrative review. Open Access J Sports Med. 2017;8:71.

16. Eberle R, Heinrich D, Kaps P, et al. Effect of ski boot rear stiffness (SBRS) on maximal ACL force during injury prone landing movements in alpine ski racing: A study with a musculoskeletal simulation model. J Sports Sci. 2017;35(12):1125-1133.

17. Heinrich D, Bogert A, Nachbauer W. Relationship between jump landing kinematics and peak ACL force during a jump in downhill skiing: a simulation study. Scand J Med Sci Sports. 2014;24(3):180-187.

18. Senner V, Michel FI, Lehner S, et al. Technical possibilities for optimising the ski-binding-boot functional unit to reduce knee injuries in recreational alpine skiing. Sports Engineering. 2013;16(4):211-228.

19. Herman K, Barton C, Malliaras P, et al. The effectiveness of neuromuscular warm-up strategies that require no additional equipment, for preventing lower limb injuries during sports participation: a systematic review. $B M C$ Med. 2012;10(1):75.

20. Koehle MS, Smith LR, Taunton JE. Alpine ski injuries and their prevention. Sports Med. 2002;32(12):785-793.

21. Mandelbaum BR, Silvers HJ, Watanabe DS, et al. Effectiveness of a neuromuscular and proprioceptive training program in preventing anterior cruciate ligament injuries in female athletes. Am J Sports Med. 2005;33(7):1003-1010.

22. Soligard T, Myklebust G, Steffen K, et al. Comprehensive warm-up programme to prevent injuries in young female footballers: cluster randomised controlled trial. BMJ. 2008;337:a2469. 